\title{
Cardiotonic activity of Pergularia tomentosa different extracts, fractions \&isolated compounds.
}

\author{
M.S., Hifnawy ${ }^{1}$, M.A. El-Shanawany ${ }^{2}$, M.M., Khalifa ${ }^{3}$ A.K, Youssef $^{4} \&$ \\ S.Y., Desoukey ${ }^{5 *}$ \\ 1 Department of Pharmacognosy, Faculty of Pharmacy, Cairo university, Egypt. \\ 2 Department of Pharmacognosy, Faculty of Pharmacy Assuit University, Egypt. \\ 3 Department of Pharmacology, Faculty of Pharmacy, Minia university, Egypt. \\ 4 Medicinal and Aromatic Plant Dept., Desert Research Center, El-Matariya, Cairo, Egypt. \\ 5Department of Pharmacognosy, Faculty of Pharmacy, Minia university Egypt.
}

\begin{abstract}
Evaluation of the cardiotonic activity of Pergularia tomentosa containing a special type of cardiac glycosides using the different aqueous extracts of the different organs (root, aerial parts \& leaves), their fractions and cardioactive isolated compounds. All were screened for toxicity testing, pharmacological \& pathological activities. A comparative study was performed on their cardiotonic activity, blood pressure, heart \& respiratory rates, ECG patterns, safety margins \& therapeutic indexes in relation to ghalakinoside-the major most active isolate. The results demonstrated dose dependent cardiotonic activity of all extracts, fractions \& isolates. The highest cardiotonic activity was produced from the major cardenolide closely followed by calactin. The most cardioactive extract was that of the roots, followed by the aerial parts \& leaves respectively. According to safety studies the aerial parts aqueous extract $\{A A E\}$ demonstrated the widest safety margin. The aqueous extract of the root demonstrated promising cytotoxic activities on some selected human tumor cell lines as cervix carcinoma (Hela cell line), liver as well as brain cell lines. The aerial parts were used for this study to conserve this plant as using the roots would mean complete destruction of the plant.
\end{abstract}

Key words:, Cardioactive, Cytotoxic, Ghalakinoside, Pergularia tomentosa aqueous extracts, Phytochemical \& Pharmacological insights.

\section{Introduction}

Cardiac disease is becoming the number one cause of death especially in civilized technological societies and there are continuous searches for new cardiotonic drugs especially from natural origins.

Pergularia is famous for a wide range of the therapeutic uses like treatment of cancers, tumors \& warts ${ }^{[1]} \&$ also contains a special type of cardenolides with doubly linked sugar moieties. ${ }^{[2 \& 3]}$. Our previous work proved the presence of these cardenolides, in lower concentrations in the aerial parts ${ }^{[4]}$. This study was performed to demonstrate the cardiotonic activity(in vivo \& in vitro) of the aqueous extracts of different organs-roots, aerial parts and leaves - in relation to the major isolate ghalakinoside along with acute toxicity studies, standard safety margins and the therapeutic indexes for each. The cardiotonic activity was measured using Langendroff's method for the determination of the \% change in force of myocardial contractions .The heart rate $\&$ also the $\%$ change in blood pressure, EGG pattern (tracing) \& respiratory rate were all measured using intact urethane anaesthetized rabbits. Previous studies demonstrated that steroidal cardenolides possess cytotoxic activity ${ }^{[5-7]}$.

\subsection{Plant Material:}

\section{MATERIALS AND METHODS}

Wild plants of Pergularia tomentosa were collected from Cairo Suez road at $27 \mathrm{Km}$ from Cairo. The plant was verified by Dr. Nahed El- Hadidy at the herbarium of the Botany Section in the Faculty of Science, Cairo University as well as by Comparison with a voucher specimen at the herbarium of the Desert Research Center (DRC).

\subsection{Extracts, Fractions \& isolates (Isolated compounds) Preparation:}

2.2.1Preparation of the dried total aqueous extracts for pharmacological and pathological studies.

Roots, aerial parts \& leaves were percolated with hot water and concentrated (1g powder/ ml) RAE $\{$ root aqueous extract\}, AAE \{aerial parts aqueous extract $\} \&$ LAE \{leaves aqueous extract $\}$. 


\subsubsection{Preparation of extracts used for toxicity study}

The dried aqueous extracts were each purified from mineral salts and sugars by dissolving in the least amount of methanol $(\mathrm{MeOH}) \&$ filtered. Filtrate completely dried and the residues were used for the toxicity study.

\subsubsection{Preparation of fraction of the aqueous extracts}

The dried purified aerial parts aqueous extract $\{\mathrm{LAE}\}$ was fractionated with column chromatography using silica gel for column, gradient elution was performed using firstly chloroform with increasing concentration of methanol, respectively. Fractions collected were monitored by TLC \& Kedde's reagent was used for visualization of cardenolide spots. Vanillin / $\mathrm{H} 2 \mathrm{SO} 4$ and 50\% MeOH/H2SO4 for other spots .Similar fractions were pooled together.

\subsubsection{Preparation of isolates}

Compounds were isolated from the major five fractions using both column and preparative TLC. These isolates were subjected to pharmacological study and spectral analysis.

Different concentration of all the above extracts, fractions and cardiotonic isolates were prepared in distilled water.

\subsection{Experimental Animals}

Healthy adult rabbits, weighing about $1.5 \pm 0.25 \mathrm{~kg}$ and adult albino mice with average weigh of $20 \pm 2$ $\mathrm{g}$ of both sexes were used with free access to food and tap water.

The animals were kept in mesh-bottomed cages with free access to food and water. Animals were fed a standard diet and water. Procedures involving animals and their care were conducted in conformity with the institutional guidelines of Minia University and it conforms to the provisions of the Declaration of Helsinki in 1995.

\subsubsection{Modified Langendorff's Apparatus}

Isolated heart perfusion apparatus was used where it permits delivery of oxygenated Lock-Ringer solution at a constant rate of $3 \mathrm{ml} / \mathrm{min}$ and temperature of $37^{\circ} \mathrm{C} .{ }^{(8)}$.

\subsubsection{Microscopical analysis}

For pathological examination of different organs, \{kidney, liver\& brain \} Paraffin sections, stained by Hematoxylin \& Eosin stain (H \& E $)$ were examined by Zeiss standard 20 microscope with Me 80 Camera (Focus 200 was used)

\subsubsection{Chromatographic analysis}

Precoated TLC plates of kieselgel F 254 (20 X 20 layer thickness of $0.2 \mathrm{~mm}$, for analysis and $2 \mathrm{~mm}$ for isolation).

\subsubsection{Solvent Systems used for TLC}

$\begin{array}{lll}\mathrm{CHCl}_{3}-\mathrm{MeOH} & 8: 2 & \text { (A) } \\ \mathrm{CHCl}_{3}-\mathrm{MeOH} & 85: 15 & \text { (B) } \\ \mathrm{CHCL}_{3}-\mathrm{CH}_{3} \mathrm{OH}-\mathrm{H}_{2} \mathrm{O} & 95: 5: 0.5 & \text { (C) } \\ \text { Ethyl acetate }-\mathrm{MeOH} & 97: 3 & \text { (D) } \\ \text { Ethyle acetate - } \mathrm{MeOH}-\mathrm{H}_{2} \mathrm{O} & 81: 11: 8 & \text { (E) }\end{array}$

Double or triple runs were performed.

\subsubsection{Reagent used for detection of spots}

1) Kedde's reagent :- Addition of equal volumes of Freshly prepared 2\% 3,5 dinitrobenzoic acid and 5\% $\mathrm{KOH}$ in Methanol

2) $\quad 2 \%$ Vanillin $/ \mathrm{H}_{2} \mathrm{SO}_{4}$

3) $50 \% \mathrm{H}_{2} \mathrm{SO}_{4}: \mathrm{MeOH}$

\subsubsection{Column chromatography C.C}

Silica gel 60 (70 - 230 mesh Merck) was used.

Column (1) $80 \times 4 \mathrm{~cm}$ gradient elution with $\mathrm{CHCl}_{3}$ with increasing amounts of $\mathrm{MeOH}$.

Column (2) $35 \times 2 \mathrm{~cm}$ gradient elution with Ethyl acetate with increasing amounts of acetone.

Techniques:

Fractionation of the dried aqueous extract 
$15 \mathrm{~g}$ of aerial parts aqueous extract of (AAE) were fractionated with $900 \mathrm{~g}$ silica gel using column (1) eluted with increasing amounts of methanol in chloroform. $250 \mathrm{ml}$ fractions was collected, concentrated to $30-40 \mathrm{ml}$ and TLC screened using system $\{$ A to $\mathrm{C}$ \} similar fractions were pooled. Kedde's reagent was used for cardenolide detection \& vanillin/ $\mathrm{H}_{2} \mathrm{SO}_{4}$ or $50 \% \mathrm{MeOH}: \mathrm{H}_{2} \mathrm{SO}_{4}$ for non-cardenolide components. Similar fractions pooled into 5 main collective fractions containing major cardenolides $\left\{A_{1}\right.$ to $\left.A_{v}\right\}$. These were redirected to further fractionation and purification.

$\mathbf{A}_{1}$ \{fraction 5-11\} eluted with $3 \% \mathrm{MeOH}$ in $\mathrm{CHCL}_{3}$ gave $2+\mathrm{Ve}$ kedde spots. These were rechromatographed on another column (2), eluted with acetone with increasing amounts of ethyl acetate where $50 \mathrm{ml}$ fractions were collected, concentrated to $5 \mathrm{ml}$ fractions, TLC screened for +Ve kedde's spots.

$\mathbf{A}_{\text {II }}$ \{fraction 12-23\} eluted with $5 \% \mathrm{MeOH}$ in $\mathrm{CHCL}_{3}$, giving 4+Ve kedde's spots. Residue passed over charcoal column $(30 \times 2 \mathrm{~cm})$ and eluted with methanol. TLC screened using Kedde\& concentrated, amorphous powder was obtained \& recrystallized from methanol.

$\mathbf{A}_{\text {III }}$ \{fraction 52-92\} eluted with $10 \% \mathrm{MeOH}$ in $\mathrm{CHCL}_{3}$ gave one + Ve kedde's spot which was concentrated \& crystals were recrystalized from methanol.

$\mathbf{A}_{\text {IV }}$ \{fraction 65-70\} eluted with $15 \% \mathrm{CH}_{3} \mathrm{OH}$ in $\mathrm{CHCL}_{3}$, TLC screening showed two +Ve kedde's spots which were directed to precoated preparative TLC for separation. The two bands separated, eluted with methanol, each eluate was concentrated \& left to crystallize.

$\mathbf{A}_{\mathbf{V}}$ \{fraction 71-90\} was concentrated and left to crystallize.

These collective fractions and isolated compounds were redirected to acute toxicity testing $\left\{\mathrm{LD}_{50}\right\}$.

\section{Biological investigations}

Pharmacological Study

The following pharmacological determinations were performed on the $\{\mathrm{RAE}\},\{\mathrm{AAE}\}$ and $\{\mathrm{LAE}\}$ and the major isolates ghalakinoside and calactin of Pergularia:-

1- Acute toxicity manifestations and the median lethal dose $\mathrm{LD}_{50}$ was calculated using Litchfiel \& Wilcoxen graphical method ${ }^{(9)}$, for the best fitting dose-response line for each of all the above tested preparation.

2- In vitro determinations of force and rate of myocardial contraction using different concentrations of the tested preparations, on the isolated perfused rabbits heart using Langendorffs method ${ }^{(8)}$ and in vivo experiments for the determination of blood pressure, respiratory rate and ECG \{Electrocardiographic\} pattern of the urethane anaesthetized rabbits.

The therapeutic indexes \& the standard safety margins were also determined for all the preparation.

\section{Cytotoxicity testing}

Potential cytotoxicity by SRB (sulforhodamine B) assay ${ }^{(10)}$ was performed at the National Cancer Institute (NCI), Cancer Biology Department, Pharmacology Unit. The relation between surviving fractions \& drug concentrations was plotted to get the survival curve of each tumor cell line after the treatment with the roots aqueous extract.

\section{Pathological examination}

Postmortem examination of mice receiving lethal doses was performed 5 minutes after respiratory arrest. Sections taken from different organs of treated animals were fixed and compared with control sections where the normal animals were receiving equal volumes of distilled water.

Microscopical examination of these fixed section were investigated.

\subsection{Fractionation}

\section{Results}

Bioassay guided fractionation of the aerial parts aqueous extract treated with methanol, revealed six cardenolides out of five $+\mathrm{Ve}$ Kedde's collective fractions. These cardenolides were identified using spectral analysis, as well as, comparing with authentic samples \{Co TLC \& Co m.p.\}and published data. These cardenolides were uzarigenin, calactin, uzarigenin-3-O-glucopyranoside desglucouarin, coroglucigenin, pergularotoside and ghalakinoside. Fig $1 \& 2$

The active cardenolides were calactin, pergularatoside and ghalakinoside, as they were isolated from active fractions AI, AIv \& Av. Further toxicity testing, pharmacological investigation and pathological studies were performed on calactin and ghalakinoside, as they were available in considerable amounts. 
<smiles>[R20]C1CCC2([R])C(CCC3C2CCC2(C)C(C4=CC(=O)OC4)CCC32O)C1</smiles>

\begin{tabular}{|l|l|l|l|}
\hline Comp. & Name & R & R1 \\
\hline 1 & Uzarigenin & $\mathrm{CH} 3$ & $\mathrm{H}$ \\
\hline 3 & $\begin{array}{l}\text { Uzarigenin-3-O- } \\
\text { Glucopyranoside }\end{array}$ & $\mathrm{CH} 3$ & Glucose \\
\hline 4 & Coroglaucigenin & $\mathrm{CH} 2 \mathrm{OH}$ & $\mathrm{H}$ \\
\hline
\end{tabular}

Fig.1<smiles>[R]C1CC2C(CCC3CC4OC5O[C@H]([2H])CC([R8])([R3])C5(O)O[C@H]4CC32[R1])C2(C)CCC(C3=CC(=O)OC3)C12O</smiles>

Fig. 2

\begin{tabular}{|l|l|l|l|l|l|l|}
\hline Comp. & Name & R & R1 & R2 & R3 & R4 \\
\hline 2 & Calactin & $\mathrm{H}$ & $\mathrm{CHO}$ & $\beta \mathrm{OH}$ & $\alpha \mathrm{H}$ & $\mathrm{CH} 3$ \\
\hline 5 & Pergularotoside & $\mathrm{OH}$ & $\mathrm{CHO}$ & $\beta \mathrm{H}$ & $\alpha \mathrm{OH}$ & $\mathrm{CH} 2 \mathrm{OH}$ \\
\hline 6 & Ghalakinoside & $\mathrm{OH}$ & $\mathrm{CH} 2 \mathrm{OH}$ & $\beta \mathrm{H}$ & $\alpha \mathrm{OH}$ & $\mathrm{CH} 2 \mathrm{OH}$ \\
\hline
\end{tabular}

\subsection{Pharmacological studies}

\subsubsection{Median lethal dose $\mathrm{LD}_{50}$}

The median lethal dose $\mathrm{LD}_{50}$ obtained from the best fitting dose-response line, revealed that the most toxic preparation was compound 6 \{ghalakinoside\}, its $\mathrm{LD}_{50}$ and 95 fiducial limits is $4.6(2.9-7.2) \mathrm{mg} / \mathrm{kg}$ followed closely by calactin of $\{5(3.367-7.425) \mathrm{mg} / \mathrm{kg}\}$ then the cardenolide fractions of the aerial parts aqueous extract $A_{V}, A_{I V}-\& A_{I}$ of $\{90(44.1-183.7) \mathrm{mg} / \mathrm{kg}, 150(126-178.5)$ and $640(340.4-621.6) \mathrm{mg} / \mathrm{kg}$, respectively.

The aqueous extracts of roots, aerial parts and leaves were $\{70(46.7-105), 147(126.4-170)$ and 680 $(557.6-829.3) \mathrm{mg} / \mathrm{kg}$, respectively.

\subsubsection{Cardiotonic activity}

In vitro pharmacological studies using Langendorffs method ${ }^{(8)}$ for the determination of force and rate of myocardial contraction using different concentrations, on the isolated perfused rabbits ' heart revealed a dosedependent positive ionotropic activity in all treatments. Ghalakinoside showed an increase in the force of myocardial contraction by a mean value of $26.7 \pm 3 \%, 49.7 \pm 0.2 \%$ and $71.0+ \pm 0.2 \%$ after the administration of $0.36,0.72$ and $1.44 \mathrm{mg} / \mathrm{kg}$ respectively and similarly calactin by a mean value of $12.8 \pm 1.2 \%, 26.6 \pm 1.6 \%$ and $23.8 \pm 0.2 \%$ after the administration of $0.5,1$ and $2 \mathrm{mg} / \mathrm{kg}$ with no change in the heart rate. The different aqueous extracts also showed this activity highest in the roots followed by the aerial parts \& lastly the leaves, where the mean increase in the myocardial contractions were of values, $50.5 \pm 1.4 \%, 109.2 \pm 0.8 \%, 67.1 \pm 0.2 \%$ and $31.7+$ $0.8 \%$ for the dose levels of 5, 10,50 and $100 \mathrm{mg}$ powder / $\mathrm{kg}$ of root aqueous extract (RAE) respectively and 28.1 $\pm 1.4 \%, 33.5 \pm 1.6 \%$ and $60.6 \pm 0.1 \%$ after the administration of dose levels of $5,10,100 \mathrm{mg}$ powder $/ \mathrm{kg}$ of aerial parts aqueous extract (AAE) respectively. Lastly the leaves aqueous extract (LAE) produced a + Ve inotropic effect of a mean value of $11.4 \pm 0.8 \%, 31.8 \pm 1.0 \%, 36.1 \pm 1.4 \%$ and $45.9+1.2 \%$ after the administration of $5,10,50$ and $100 \mathrm{mg}$ powder / $\mathrm{kg}$ of leaves aqueous extract (LAE).

The heart rate was affected at very high dose levels of $100 \mathrm{mg}$ powder / $\mathrm{kg}$ for the root and the leaves aqueous extracts. A slight -Ve chronotropic effect was demonstrated but it was decreased at a lower dose level of $10 \mathrm{mg}$ powder / $\mathrm{kg}$ for the aerial parts aqueous extract. The in-vivo experiments for the determination of blood pressure and respiratory rate of urethane anaesthetized rabbits demonstrated that all the treatments showed variable changes in the mean arterial blood pressure which were reversible except at the lethal dose of calactin. The ghalakinoside showed a mean $\%$ change of $35 \pm 4.5 \%, 55 \pm 7.8 \%, 80 \pm 9.4 \%$ and $81.3+4.0 \%$ for dose levels of $0.36,0.72,1.2$ and $3.6 \mathrm{mg} / \mathrm{kg}$, while calactin demonstrated mean changes of $16 \pm 0.7 \%, 30 \pm 0.8 \%$ and $128 \pm 3.1$ $\%$ for dose levels of 1.0, 2.0 and $6 \mathrm{mg} / \mathrm{kg}$. The last dose is considered a lethal dose which causes severe 
hypotension for the aqueous extracts; dose levels of 5, 10, 20, $50 \& 100 \mathrm{mg}$ powder/kg were tested. A mean $\%$ change in the arterial blood pressure of $19 \pm 1.7 \% \& 35 \pm 2.3 \%$ was observed at a dose level of $50 \mathrm{mg}$ and 100 $\mathrm{mg}$ powder/kg of (RAE) root aqueous extract, higher doses cause hypotension $\&$ decreased respiratory rate. The above dose levels of the aerial parts aqueous extract (AAE) revealed a mean \% change in the arterial pressure of $17 \pm 3.3 \%$ and $43 \pm 8 \%$ respectively, while for the leaves aqueous extract (LAE) the dose levels of $100 \& 150$ $\mathrm{mg}$ powder $/ \mathrm{kg}$ revealed a mean $\%$ change of $10 \pm 1.4 \%$ and $39 \pm 0.2 \%$.

\subsubsection{Electrocardiographic (ECG) Changes}

ECG patterns of the same urethane anaesthetized rabbits were studied after treatment with the two isolates Ghalakinoside \& calactin and the three previously mentioned aqueous extracts. There was no change observed in the heart rates, only mild reversible bradycardia with high dose levels. Bradycardia was observed with doses of $1.2 \& 3.6 \mathrm{mg} / \mathrm{kg}$ of ghalakinoside, higher doses above $12 \mathrm{mg} / \mathrm{kg}$ demonstrated the same action with irreversible changes in heart rate \& ECG pattern. Higher doses of ghalakinoside $(24 \mathrm{mg} / \mathrm{kg}) \&$ calactin $(6$ $\mathrm{mg} / \mathrm{kg}$ ) showed changes in cardiac rhythm (dysrythmia) ended with a heart block. The aqueous extracts exerted reversible bradycardia at high dose levels of $50,100 \& 150 \mathrm{mg} / \mathrm{kg}$ powder $/ \mathrm{kg}$ for RAE, AAE \& LAE, respectively.

\subsection{Pathological examination:}

Postmortem examination of mice receiving lethal doses \{One gm/kg for Ghalakinoside \& $2,4 \& 2 \mathrm{~g}$ powder $/ \mathrm{kg}$ for RAE, AAE \& LAE, respectively $\}$ was performed. Upon opening the chest of these treated (injected) animals, all treatments showed more or less the same picture. Congestion was obvious in many organs. Both lungs appeared voluminous, soft, and pink with rounded borders. The heart showed mild enlargement and softness with dilated chambers. The liver was increased in size with rounded borders \& soft consistency with deep red colouration. Both kidneys were markedly congested. No motility - was observed in the gastrointestinal tract (GIT). The intestine and stomach were both congested. Opening the skull revealed congested meninges \& brain hemorrhage.

Microscopical examination of fixed section showed clear congestion in all fixed slides. The lungs showed vasodilation foci of acute pulmonary oedema \& hemorrhage with little blood cells in alveolar space. While the liver showed congested central veins \& sinusoids but the brain was not affected.

Severity of symptoms was greatest in these toxic doses of ghalakinoside followed by the aqueous extracts of roots, aerial parts \& leaves respectively, but all were reversible.

\subsection{Toxicity study:}

Acute toxicity manifestations revealed generalized dose dependent depression of CNS higher doses caused sedation, followed by loss of their spontaneous searching out activity with regular breathing \& tackycardia, animals move with difficulty and regain their normal activity after a certain period of time. Toxic doses cause the above symptoms immediately after injection followed by rapid loss of skeletal muscle tone with some convulsive fits. Muscle paralysis followed, starting from hind then to fore limbs. The head dropped \& animals became ataxic with a waddling gate, abdomen sagged \& touched the ground. Irregular respiration terminated with respiratory arrest \& tackycardia. Cyanosis was also observed specially in non-hairy parts of tail, ears \& mouth. These symptoms were more prominent in ghalakinoside \& calactin.

\subsection{Safety studies}

The therapeutic indexes \& the standard safety margin were determined for each of the aqueous extracts in comparison with isolated compounds. The therapeutic index was highest for RAE followed by AAE, LAE \& finally ghalakinoside. According to the study highest standard safety margin was shown with AAE followed by LAE \& RAE and the least was of Ghalakinoside.

\subsection{Cytotoxic studies}

Cytotoxicity testing gave promising results for RAE using SRB assay. Where IC50 of RAE was $7.5 \mu \mathrm{g} / \mathrm{ml}$ for Hela cells of cervix carcinoma, $9.97 \mu \mathrm{g} / \mathrm{ml}$ for Hepg 2 liver carcinoma \& closely followed by $10 \mu \mathrm{g} / \mathrm{ml}$ for U251 brain tumor cell line.fig. 3 


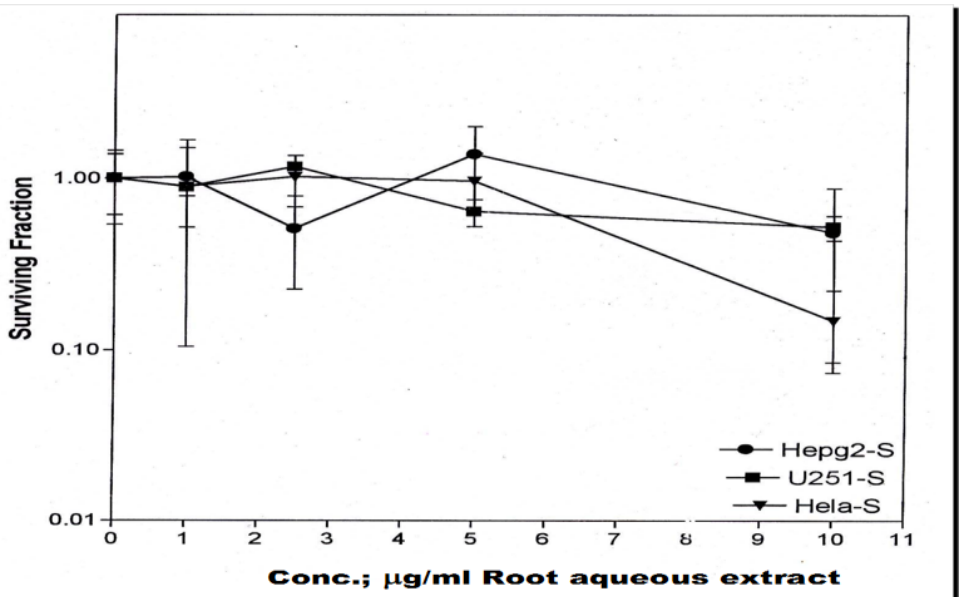

Fig. 3 The surviving fraction of the human cell lines listed against the root aqueous concentration in $\mu \mathrm{g} / \mathrm{ml}$.

\section{Discussion}

The pharmacological activity of the aqueous extracts of the roots, aerial parts \& leaves (RAE, AAE \& LAE) of Pergularia is due to the presence of three cardioactive water soluble, cardenolides: calactin, ghalakinoside \& pergularatoside. Bioassay guided fractionation of the aerial parts aqueous extracts (AAE) resulted in 5 main cardioactive fractions. Upon isolation \& purification of each fraction, the same active cardenolides of the RAE were obtained (but in lower concentrations). Using the aerial parts would conserve this biologically important Egyptian plant and prevent its destruction as would be the case when using its roots. The comparative study for the three aqueous extracts with the major cardenolide, ghalakinoside using toxicity testing, pharmacological activities on in vivo \& in vitro cardiovascular system and pathological examinations demonstrated the wide safety of the three extracts. The highest therapeutic index was for RAE followed by the AAE \& LAE and lastly the major isolate ghalakinoside, while the widest standard safety margin was that of AAE followed by the LAE then the RAE and lastly pharmacologicaly, the extracts had similar effect to the isolated cardenolides, and the side effects observed as the change in the arterial blood pressure and heart or respiratory rates were only observed at lethal doses, much higher than those required for cardiotonic activity. Pathological examination revealed that the 3 extracts and the ghalakinoside had no effects on the brain cells, although they functionally affected the CNS at lethal doses. The degenerative effects on lungs, liver \& kidneys were all reversible and non destructive, although comparatively, the most severe results were demonstrated by the isolated compound ghalakinoside. The roots aqueous extract (RAE) was examined for cytotoxicity using Erlich acytic carcinoma (EAC) and some human tumor cell lines. Promising results on the three tested tumor cell lines were demonstrated at the chosen concentrations for the roots aqueous extract. The highest activity was achieved with Hela cell line, where the extract caused inhibition of cervix carcinoma cells to $50 \%$ with a dose of $7.5 \mu \mathrm{g} / \mathrm{ml}$, followed by Hepg 2 (liver carcinoma) where IC50 was $9.97 \mu \mathrm{g} / \mathrm{ml}$ and lastly the U 251 brain tumor cell line had an $\mathrm{IC}_{50}$ of $10 \mu \mathrm{g} / \mathrm{ml}$.

\section{Conclusion}

The mentioned results demonstrate that the aerial parts could be used as a cardiotonic agent and also proved a wider safety margin, from this study we could also conserve this plant which is liable to extinction as many medicinal desert plants, as using the roots would mean complete destruction of the plant.

\section{References}

[1] Kupohan, S.M., Knox, J.R., Kelsey, J.E. and Renauld, J.A. (1964)Science 146.

[2] Cheung, H.T.A. and Watson, T.R. (1980). J.C.S. Perkin I P.2162-2168. Steriochemistry of the hexosulose in cardenolide glycosides of Asclepiadaceae.

[3] A1 Said, M.S.; Hifnawy, M.S.; Mc phail, A.T. and Mc Phail, D.R. (1988). Phytochemistry: 27(10) P.3245 - 3250. ghalakinoside a cytotoxic cardiac glycoside from Pergularia tomentosa.

[4] Desoukey, S.Y.; Hifnawy, M.S., El-Hawary, S.S. and Amer, Kh.F.(1998). MSc. thesis, Faculty of Pharmacy, Cairo University.

[5] Mc Conkey D.J., Orrenius, S. (1997). Biochem. Biophys. Res. Commun,: 239 P. 357-366 (The role of Calcium in regulation of apoptosis).

[6] Mc Conkey, D.J.; Yun Lin, Nutt, L.K.; Ozel, H.Z. and Newman, R.A. (2000). Cancer Research: 60 (14) p. 3807-3812. (Cardiac glycosides stimulate $\mathrm{Ca}^{2+}$ increase and apotosis in androgen independent, metastatic human prostate Adenocarcinoma cells).

[7] Stenkvist, B. (1999). Oncol. Rep.: 6 p.493-496 (Is digitalis a therapy for breast carcinoma).

[8] Langendroff, O. Untersuchungen am uberlebenden saugethierherzen. Pfluger Arch. 61: 291-332, 1895 (European journal of physiology) Springer link.

[9] Litchfield, J.T., Jr and Wilkonoxon, F. (1948). J. Pharmacol. \& Exp. Therap. :96 p. 99-113 
[10] Skehan, R. Storeng, D. Scudiero, A. Monks, J. McMahon, D. Vistica, J.T. Warren, H. Bokesch, S. Kenney, and M.R. Boyd (1990). J Nat Cancer Inst.: 82 P.1107-1112 (New colorimetric cytotoxicity assay for anticancer-drug screening).

[11] Vogal's (1978) fifth Edition, B.S.Furniss, et al "Vogal's text book of practical organic chemistry" E.L.B.S., London, Fuchs, P.spiser, Pharmaceutical Technology) Gerory thieme Berlag, Stuffgont, 1978.

[12] Vyas, G.N. and Shal, N.M. (1963). Organic synthesis: Collective volume 4 p. 836 (checked by Williams Jonhson and R1.T. Keller\}. 